\title{
Experiencia metodológica en la capacitación de promotores de seguridad vial en México
}

\section{A methodological experience in the training of road safety promoters in Mexico}

DOI: $10.32870 /$ dse.v0i19.519

\section{Luis David Berrones-Sanz*}

\begin{abstract}
Resumen
Este trabajo documenta la experiencia de formación de promotores de seguridad vial, que se realizó como propuesta para el componente de capacitación del modelo de intervención para reducir los accidentes de tránsito de la Iniciativa Mexicana de Seguridad Vial (IMESEVI). La planeación del curso-taller se desarrolló en cuatro etapas: 1) documentación, 2) diseño, 3) operacionalización y, 4) seguimiento y evaluación. Entre el año 2010 y 2012 se realizaron ocho cursos-taller en siete estados de la república mexicana (Ciudad de México, Estado de México, Guanajuato, Michoacán, Puebla, Tamaulipas y Veracruz), fueron impartidos por dos especialistas (en promoción de la salud y en ingeniería en transporte) y posteriormente sistematizados para identificar generalidades y particularidades locales o regionales que aportaran elementos para el ajuste del plan de intervención y del material didáctico. La instrumentación, de forma modular, se sustentó en el aprendizaje a partir de problemas detectados en la experiencia privilegiando la reflexión y acción, de tal manera que los formadores replicaran las mismas herramientas pedagógicas en sus propios talleres. La perspectiva y los contenidos temáticos se impartieron con un enfoque técnico, pedagógico y de salud, con la intención de comprender las conductas que denotan la reproducción de prácticas de riesgo y, aunque permitieron compartir experiencias, reflexiones y seguir cuestionándonos, sensibilizándonos y recreándonos juntos, no han demostrado su eficiencia empírica ya que ha sido mínima su incidencia en la disminución de accidentes viales.
\end{abstract}

Palabras claves: Promoción de la salud - aprendizaje basado en problemas - taller de reflexiónacción - seguridad vial.

\begin{abstract}
This paper documents an experience in the training of road safety promoters conducted as a trial training workshop of a model intervention to reduce the number of road accidents in the Mexican Road Safety Initiative (Iniciativa Mexicana de Seguridad Vial, IMESEVI). The workshop was planned in four stages: 1) literature review, 2) design, 3) operationalization and 4) monitoring and evaluation. Between 2010 and 2012, eight workshops were held in seven states of the Mexican Republic (Mexico City, Mexico State, Guanajuato, Michoacán, Puebla, Tamaulipas and Veracruz), taught by two specialists in health promotion and transportation engineering, and subsequently,
\end{abstract}

* Doctorado en Ciencias Administrativas. Universidad Autónoma de la Ciudad de México. Escuela Superior de Comercio y Administración, Instituto Politécnico Nacional. México. luis.berrones@uacm.edu.mx 
systematized to identify general and specific local or regional characteristics that provided data to customize the intervention plan and teaching materials. The modular implementation was executed using a problem and experience-based learning approach. A reflection-action methodology was emphasized, allowing trainers to use the same teaching tools in their own workshops. The focus of the approach and contents was on technical, pedagogic and health issues, aiming at understanding behaviors leading to risk practices. Even though the workshops promoted the sharing of experiences, thoughts, and questionings, this work has not provided empirical results, since there has been minimal reduction of road accidents.

Keywords: health promotion - problem-based learning - reflection-action workshop - road safety.

\section{Introducción}

En el año 2007, la Organización Mundial de la Salud (OMS), la Organización Panamericana de la Salud (OPS) y los gobiernos de México, Vietnam y Camboya, establecieron acuerdos para realizar programas sobre seguridad vial. De ahí que la Secretaría de Salud, a través del Centro Nacional para la Prevención de Accidentes (CENAPRA), construyera la Iniciativa Mexicana de Seguridad Vial (IMESEVI) con el objetivo de reducirlas 750,000 hospitalizaciones y 20,000 muertes de personas por año en México por motivo de accidentes. Para esto, se estableció un modelo de intervención basado en evidencia científica, con un enfoque integral y un abordaje multisectorial.

Uno de los componentes del modelo de intervención, pensado para sensibilizar a la población sobre la gravedad del problema y las conductas a seguir, es la capacitación; ésta se basa en un sistema piramidal en la que dos formadores reproducen la información a 20 multiplicadores y estos, a su vez, deberán capacitar a 150 personas de la población en general. Con esta finalidad, la IMESEVI elaboró un manual de orientaciones básicas que abarcan seis factores de riesgo en la seguridad vial (la velocidad, el uso del cinturón de seguridad, los sistemas de retención infantil, el consumo de alcohol, los peatones y el uso de casco) teniendo como hilo conductor la formación y la educación.

De esta forma, la capacitación de los formadores, inicio de la pirámide y de la estructura de capacitación (STCONAPRA, 2011), requiere herramientas pedagógicas y técnicas que den bases sólidas y permitan la trasmisión del conocimiento; para esto se propuso el curso de formadores en seguridad vial; el cual es construido con una visión integral, a partir del análisis de investigación y experiencia de cada uno de los autores.

La perspectiva del curso y los contenidos temáticos se impartieron con un enfoque técnico, pedagógico y de salud, que permite comprender las conductas que denotan la reproducción de prácticas de riesgo; pretende desarrollar habilidades pedagógicas y didácticas necesarias para la intervención en grupos y que permita diseñar estrategias de intervención contextualizadas, orientadas a la prevención de accidentes, que considere las necesidades y características de la población meta y sus prácticas sociales.

Diálo@os sobre Educación año 10 | número 19 | julio-diciembre 2019 | ISSN 2007-2171 
De aquí que este artículo tiene como propósito documentar la experiencia de capacitación y formación de promotores en seguridad vial con una perspectiva de la promoción de la salud de talleres de reflexión-acción en los que, durante cerca de un año, un equipo de la Universidad Autónoma de la Ciudad de México participó impartiendo ocho cursos en diferentes partes de la república mexicana y en los que se capacitó a 214 formadores de seguridad vial.

\section{Marco teórico}

El Aprendizaje Basado en Problemas (ABP) es un modelo pedagógico desarrollado en la década de los sesenta por el programa de Medicina de la Universidad de McMaster en Hamilton, Ontario, Canadá; en la que de acuerdo con Barrows (1986), uno de los creadores, este modelo busca que los estudiantes adquieran e integren nuevos conocimientos a través de la experiencia de resolución de problemas. Sánchez S. y Ramys (2004) establecen que el ABP incentiva a los estudiantes en el desarrollo de competencias laborales para trabajar en equipos de investigación, produciendo y adquiriendo habilidades colectivas de comunicación e integración de información. Entre las características principales de la ABP, Barrows (1996) menciona las siguientes:

1. El aprendizaje está basado en el estudiante. Es decir, los estudiantes deben tomar la responsabilidad de su propio aprendizaje de manera personalizada y de acuerdo a su área de interés.

2. El aprendizaje se produce en grupos pequeños de estudiantes. El trabajo debe ser intenso y en grupos de cinco a diez personas, que deben estar en cambio constante.

3. Los facilitadores o tutores guían a los estudiantes en lugar de enseñar. No se debe proporcionar información directa a los estudiantes, sino plantear cuestionamientos que ayuden a las personas a entender y manejar los problemas por sí mismas.

4. Un problema constituye la base para el enfoque organizado del grupo y para estimular el aprendizaje. Los problemas deben representar un desafío y motivar a los estudiantes para la integración de varias disciplinas que permitan aplicar lo aprendido en futuros problemas.

5. Los problemas son un vehículo para el desarrollo de habilidades de resolución de problemas y estimulan el proceso cognitivo. Por lo que los problemas deben ser lo más parecido a situaciones reales a las que el estudiante se enfrentará en su vida profesional.

6. El nuevo conocimiento se adquiere aprendizaje autodirigido. Los integrantes del grupo deben revisar, discutir y debatir de manera permanente los conocimientos adquiridos.

Para diseñar clases con el modelo de ABP, Morales y Landa (2004) señalan que primero se deben tener los objetivos de aprendizaje y posteriormente establecer el tiempo de duración, la forma de evaluar y el proceso a seguir. Además, estas autoras sintetizan la ruta que siguen los 
estudiantes durante el desarrollo del proceso de ABP en los siguientes ocho puntos: 1) leer y analizar el escenario del problema, 2) realizar una lluvia de ideas, 3) hacer una lista de aquello que se conoce, 4) hacer una lista de aquello que se desconoce, 5) hacer una lista de lo que necesita hacerse para resolver el problema, 6) definir el problema, 7) obtener información, y 8) presentar resultados.

Actualmente, el modelo ABP es utilizado no sólo en las ciencias de la salud, donde fue empleado por primera vez, sino prácticamente en todos los campos del conocimiento (administración, derecho, matemáticas, ingeniería, entre otras) (Cotič y Zuljan, 2009; Echevarría, 2010; Gorbaneff, 2010); al igual que otros modelos pedagógicos centrados en el estudiante, el ABP ha sido motivado por el reconocimiento de los fracasos de la enseñanza tradicional (Kenney, 2012) y el surgimiento de una comprensión más profunda de cómo la gente aprende. Sin embargo, a diferencia de la enseñanza tradicional, se enfrenta a la naturaleza del saber mismo, que se apoya sobre la experiencia e intuición de los instructores, por lo que el docente prefiere evitar este método (Gorbaneff, 2010). No obstante, el ABP involucra activamente al estudiante en un proceso, en el que se incluyen problemas que pueden resolverse de maneras diferentes y que tienen más de una solución, dando como resultado un método exitoso y significativo para la construcción de conocimiento.

\section{Descripción metodológica}

Se formó un grupo transdisciplinario compuesto por integrantes de las academias de las licenciaturas de Promoción de la Salud y de Ingeniería en Sistemas de Transporte Urbano de la Universidad Autónoma de la Ciudad de México (UACM). Se planteó que para remediar los problemas actuales relacionados con el transporte, la salud, la seguridad y la educación vial, es imperiosa la intervención de profesionistas de diferentes áreas de conocimiento. Por tal motivo, la propuesta de trabajo fue sustentada en la transdisciplinaridad a fin de analizar y caracterizar de manera integral los objetos de estudio; asimismo, podrían proponerse diferentes y mejores alternativas de solución como producto de las relaciones de colaboración y cooperación mutua, mediante las aportaciones de los distintos saberes y experiencias de profesionistas formados en diversas disciplinas. Cada problemática se aborda desde la perspectiva de la sociología urbana y del trabajo, la ingeniería de transporte, la salud pública y la pedagogía, sin dejar fuera los aspectos económicos, ambientales y organizacionales.

\section{Descripción de la experiencia}

La planeación del curso-taller se desarrolló en las cuatro etapas subsecuentes:

\section{1) Documentación}

La etapa de documentación consistió en revisar el estado de conocimiento sobre seguridad vial y en la elaboración de un diagnóstico analítico. Para el primer punto, se abordaron las investigaciones, programas y campañas nacionales e internacionales para la prevención de accidentes, particularmente las experiencias de intervención exitosas. Mientras que el diagnóstico analítico

Diálo@os sobre Educación año 10 | número 19 | julio-diciembre 2019 | ISSN 2007-2171 
se construyó a partir de los resultados de la medición de los factores de riesgo en materia de seguridad vial, así como de las estadísticas de los accidentes de tránsito terrestre del Instituto Nacional de Estadística y Geografía (INEGI) y las buenas prácticas ya documentadas por IMESEVI y CENAPRA.

\section{2) Diseño}

En la etapa de diseño se utilizó la información de la etapa de documentación, y tomando en cuenta el perfil de los participantes potenciales (personal de tránsito y protección civil, médicos, paramédicos y personal de salud, entre otros) se diseñó el curso curso-taller y las cajas de herramientas para los replicadores. El producto de esta etapa fue una carta descriptiva, muy detallada, que contiene los objetivos, las actividades, las dinámicas, los materiales y el tiempo de duración de cada actividad. En esta etapa se realizaron sesiones de práctica con estudiantes de la UACM para validar el tiempo y encontrar áreas de oportunidad; se elaboró el material de apoyo y proyección, y se consiguieron los materiales, dispositivos e instrumentos necesarios para su operacionalización. El contenido temático se basó en los manuales y material multimedia que, previamente, el CENAPRA había preparado con la información básica del programa y de los factores de riesgo (STCONAPRA, 2011). Así, utilizando estas herramientas, el curso-taller fue elaborado a partir del aprendizaje basado en problemas y experiencias, privilegiando la reflexión-acción, de tal manera que los formadores pudieran replicar las mismas herramientas pedagógicas en sus propios talleres.

\section{3) Operacionalización}

Esta etapa se dividió en tres partes: a) taller de formación y selección de replicadores, b) análisis de la experiencia, y c) talleres de replicadores.

\section{Taller de formación y selección de replicadores}

Esta primera subetapa tenía los propósitos de obtener propuestas para la evaluación y ajuste de la caja de herramientas (toolkit) que los replicadores podrían utilizar en sus talleres; desarrollar habilidades para la intervención grupal en talleres; ajustar el perfil de replicadores del taller y seleccionar los participantes que cumplieran con el perfil.

Los contenidos de los talleres se organizaron en módulos y cada factor de riesgo (conducir bajo la influencia del alcohol, velocidad inadecuada, no uso de cinturón de seguridad, no uso de cascos y sistemas de retención infantil) constituyó un módulo. Además, se pensó que la organización por módulos también permitiría la jerarquización de los factores de riesgo, considerando la percepción de los participantes, clase social u otros datos sociodemográficos. De ahí que el diseño modular permitiría que, en la réplica de los talleres, los módulos se organizaran con base en la jerarquización proporcionada por cada grupo de participantes. 
En cuanto a la estrategia de intervención, se buscó una problematización colectiva con cada uno de los factores de riesgo y, a partir de las experiencias de los participantes, describir de manera colectiva las situaciones, las prácticas y los hábitos relacionados con los esquemas de percepción y representación vinculados a los comportamientos de riesgo; además de informar sobre datos relacionados con las consecuencias de cada uno de los factores de riesgo en la seguridad personal y de otros; reflexionar sobre las representaciones (resignificar) de los participantes acerca de factores de riesgo y factores protectores; construir propuestas para transformar los riesgos en factores protectores; y desarrollar habilidades para la intervención en grupos.

\section{Análisis de la experiencia}

En el análisis de la experiencia se planteó la sistematización de los productos de los talleres para identificar generalidades y particularidades locales o regionales que aportaran al ajuste del plan de intervención y del material correspondiente (manuales para replicadores y material para los talleres).

\section{Talleres de replicadores}

Se pensaba la aplicación de talleres con la moderación de los replicadores y la participación de un responsable del taller y un observador externo. Por lo que se tendrían como productos los talleres de réplica en otras poblaciones, más replicadores y elementos para el diseño de campañas publicitarias.

\section{Seguimiento y evaluación}

En esta etapa se planeó el monitoreo a los talleres mediante la elaboración de reportes que los replicadores tendrían que generar, así como observaciones directas o a través de videos que permitieran el mejoramiento de los cursos-talleres.

Para la evaluación, se propusieron tres horizontes:

1. Corto plazo: donde se planteaba una valoración de los participantes en los talleres, identificando los conocimientos adquiridos y las técnicas didácticas.

2. Mediano plazo: se planeaba evaluar y sistematizar datos sobre la evolución y permanencia del programa.

3. Largo plazo: con la propuesta de evaluar el programa a través de la medición de incidencia y mortalidad por accidentes de tránsito, así como el incremento en la presencia de factores protectores para la seguridad vial, recuperando la metodología desarrollada por IMESEVI para el diagnóstico. 


\section{Ejecución del curso-taller}

Entre los años 2010 y 2012, en total se realizaron ocho cursos en siete estados de la república mexicana (tabla 1), cada uno con duración de 56 horas. En promedio participaron 26.6 personas por curso, con un total de 214 , de las cuales $67 \%$ eran de sexo masculino. El grupo más grande (48.4\%) pertenecía a fuerzas policiacas (como seguridad pública o tránsito municipal); le sigue, con $41.3 \%$, el personal de la Secretaría de Salud (organizadores de los cursos), principalmente los responsables de los programas de prevención de accidentes de las diferentes jurisdicciones estatales; personal de centros de salud (5.6\%), protección civil (1.9\%), Secretaría de Educación (1.4\%) y organizaciones no gubernamentales (1.4\%); por ello, las profesiones fueron muy variadas: oficiales de policía, médicos, enfermeros, técnicos en urgencias médicas, bomberos, licenciados, contadores, administradores y hasta arquitectos.

Se realizaron dos cuestionarios con 14 reactivos, uno a manera de diagnóstico, al inicio del curso, y otro para evaluar, al final del curso. Los reactivos fueron de opción múltiple y se preguntaba acerca del sistema nacional de capacitación del IMESEVI y de los factores de riesgo en la seguridad vial. Las evaluaciones (tabla 1) mostraron mejoría en cuanto a adquisición de conocimiento, pero lo más importante es que cada participante preparó material para reproducir un curso o platica para divulgar y sensibilizar acerca de la importancia de la seguridad vial en diferentes tipos de poblaciones. Así, por ejemplo, hubo quienes planearon pláticas para adultos mayores, para madres de neonatos, niños y jóvenes universitarios. Cada participante escogió su población meta, de acuerdo a su área laboral, experiencia o necesidades regionales. Además, expusieron su proyecto al grupo y el límite fue hasta donde la creatividad alcanzara; muchos reprodujeron actividades de los instructores de la UACM, en la que, incluso, se ofrecían bebidas alcohólicas a voluntarios, para posteriormente hacer la medición del nivel de alcohol con un alcoholímetro, y discutir acerca de las diferencias metabólicas; otros prepararon obras de teatro y hasta actuaciones de payasos.

\section{La dinámica de las sesiones}

Al iniciar cada sesión, se formaron grupos cercanos a las cinco personas, que participaron como equipo o como núcleo inicial de discusión. Frente al grupo siempre hubo dos instructores, un moderador y uno de apoyo. El primero se encargaba de dar las instrucciones de las actividades a realizar en cada sesión, planteaba el problema, verificaba que todos entendieran el objetivo, motivaba la participación de todos por igual, asignaba los turnos y controlaba el tiempo; el segundo instructor, a manera de lluvia de ideas, tomaba nota de las aportaciones, los conocimientos y las experiencias previas de los miembros de cada equipo, de tal manera que se tuviera cierto grado de organización. Cada grupo de participantes debía realizar las actividades y solucionar los problemas que se planteaban utilizando cualquier recurso disponible, como el material de apoyo o realizando investigaciones en Internet. Así, en un momento subsecuente, 
cada equipo podía comenzar a hacer conclusiones o replantearse la investigación, de tal manera que se pudiera proceder a valorar las soluciones planteadas.

Tabla 1. Número de participantes y promedios de las pre y las pos-evaluaciones

\begin{tabular}{|c|c|c|c|c|}
\hline \multirow{2}{*}{ Estado } & \multicolumn{2}{|c|}{ Pre-evaluación } & \multicolumn{2}{|c|}{ Pos-evaluación } \\
\hline & $\mathbf{n}$ & $(M \pm D S)$ & $\mathbf{n}$ & $(M \pm D S)$ \\
\hline Ciudad de México & 38 & $(6.80 \pm 2.10)$ & 38 & $(9.02 \pm 0.54)$ \\
\hline Estado de México & 18 & $(6.63 \pm 1.80)$ & 18 & $(8.49 \pm 0.69)$ \\
\hline Guanajuato (Silao) & 27 & $(6.75 \pm 1.94)$ & 27 & $(9.05 \pm 0.63)$ \\
\hline Guanajuato (León) & 32 & $(6.34 \pm 1.80)$ & 32 & $(8.71 \pm 0.56)$ \\
\hline Michoacán & 18 & $(6.79 \pm 2.11)$ & 18 & $(8.89 \pm 0.44)$ \\
\hline Puebla & 40 & $(6.13 \pm 1.92)$ & 40 & $(8.96 \pm 0.70)$ \\
\hline Tamaulipas & 26 & $(7.09 \pm 2.02)$ & 26 & $(8.82 \pm 0.57)$ \\
\hline Veracruz & 14 & $(6.48 \pm 1.85)$ & 14 & $(8.83 \pm 0.60)$ \\
\hline Total & 214 & $(6.57 \pm 1.99)$ & 214 & $(8.83 \pm 0.86)$ \\
\hline
\end{tabular}

$\mathrm{n}=$ número de participantes. $\mathrm{M}=$ Media de la evaluación.

DS = Desviación estándar de la evaluación

\section{Consideraciones sobre la metodología}

Las evaluaciones a corto plazo indicaron que el objetivo se cumplía, se adquirían los conocimientos básicos a través de problemas, y los participantes diseñaban y presentaban un taller, que replicarían posteriormente.

A mediano plazo, se puede observar la continuidad del programa; el Secreatriado Técnico del Consejo Nacional para la Prevención de Accidentes (STCONAPRA) indica que hasta el año 2017 se contaba con 33,206 promotores en seguridad vial, en 27 entidades federativas, por lo que ya se ha sensibilizado a 4'452,282 personas en el tema de seguridad vial. Además de que ya se tienen equipos de formadores en cerca de 80\% del territorio nacional STCONAPRA, 2018).

No obstante, si bien los formadores adquieren conocimientos y herramientas prácticas y pedagógicas para sensibilizar acerca de la seguridad vial, el efecto en la reducción de accidentes es muy limitado, ya que la población sensibilizada no representa ni dos puntos porcentuales de la población total del país. Por lo que el curso-taller es efectivo como instrumento de capacitación del personal de las áreas involucradas en la prevención de accidentes, pero está muy limitado como estrategia nacional de reducción de accidentes.

\section{Conclusiones}

La experiencia en Aprendizaje Basado en Problemas ha sido rica para compartir experiencias, reflexiones y para seguir cuestionándonos, sensibilizándonos y recreándonos juntos. Los cursos-taller permitieron enriquecer las propuestas de formación de promotores de seguridad vial, 
además de que facilitaron la conexión de los aspectos teóricos con los de la práctica profesional. Así, los instructores se convierten en facilitadores, y el aula, en una colectividad de aprendizaje que busca el conocimiento mediante cuestionamientos compartidos.

Sin embargo, los cursos-taller no han demostrado su eficiencia empírica ya que parece no haber seguimiento al grupo de formadores. El componente de capacitación del Programa de Seguridad Vial es difícil de evaluar en cuanto sus resultados en la disminución de accidentes viales; además de que la evaluación a largo plazo no se ha podido realizar debido a cambios en la estructura organizacional o falta de participación de los formadores.

Por un lado, el STCONAPRA (2018) indica haber capacitado a más de 33 mil promotores, por lo que evidentemente ha contribuido de manera sustancial en la capacitación sobre seguridad vial de los profesionales de la salud, pero no se muestra claramente qué porcentaje de los responsables de la prevención de accidentes ha participado en los cursos de capacitación. Se menciona que se ha involucrado a cerca de $80 \%$ de las entidades federativas, pero el total de promotores es un acumulado a lo largo de cerca de ocho años, y no se refiere a un corte transversal que nos permita conocer la proporción del personal que ha sido capacitado y que ejecuta el programa en un periodo determinado. Por lo que el STCONAPRA tiene que realizar el seguimiento de los formadores $y$, constantemente, reimpartir los talleres -incluyendo a los mismos formadores- para evaluar y reforzar los conocimientos sobre la seguridad vial y el programa de prevención de accidentes; compartir las buenas prácticas ejercidas durante la sensibilización de la población meta y retomar la aplicación de la experiencia metodológica. No obstante, la capacitación y actualización de formadores no influirá directamente en la disminución de accidentes, pero permitirá una mejor toma de decisiones para diseñar, implementar y ejecutar los programas regionales de seguridad vial.

Por otro lado, el indicador 4 del Programa de Acción Específico 2013-2018 de la Secretaría de Salud (2014), referente a la población sensibilizada por los promotores de seguridad vial, señala que para el año 2017, 1.4 millones de personas entre 10 y 49 años de edad, recibieron una plática sobre seguridad vial. Si bien es cierto que el STCONAPRA (2017) sobrepasó la meta, dando pláticas a $1.87 \%$ de la población, y que se puede inferir la importancia de dar a conocer y promover la adopción de conductas seguras en los usuarios de las vialidades mediante la capacitación y sensibilización de la población, no se sabe cuál es la verdadera incidencia de este indicador en la reducción de accidentes. Así, para encontrar la Relación de Riesgo habría que conocer la cantidad de personas que recibieron capacitación o sensibilización que estuvieron involucradas en algún siniestro. El tema es complicado debido a que durante las pláticas de seguridad vial, los participantes o sus tutores deberían firmar un consentimiento informado y proporcionar algún identificador oficial -como la Clave Única de Registro de Población- para que, posteriormente, el personal de la Secretaría de Salud realice el análisis -en total confidencialidad y de acuerdo al cuidado de datos personales- y que, con los datos de mortalidad se 
pueda hacer el cruce de variables, obtener el factor de exposición de la población sensibilizada, y así poder evaluar de manera cuantitativa la eficiencia del programa.

En suma, se tienen que dirigir esfuerzos para ligar las experiencias de educación con los efectos en las tasas de accidentes. Por lo pronto, lo que se muestra es que no hay manera de demostrar que la capacitación incide en el comportamiento de los usuarios de las vialidades.

\section{Referencias}

Barrows, H. S. (1986). "A Taxonomy of Problem-Based Learning Methods". Medical Education, 20(6), 481-486. https://doi.org/10.1111/j.1365-2923.1986.tb01386.x

(1996) "Problem-Based Learning in Medicine and Beyond: A Brief Overview". New Directions for Teaching and Learning, (68), 3-12. https://doi.org/10.1002/tl.37219966804

Cotič, M. y M. V. Zuljan (2009). "Problem-Based Instruction in Mathematics and its Impact on the Cognitive Results of the Students and on Affective - Motivational Aspects". Educational Studies, 35(3), 297-310. https://doi.org/10.1080/03055690802648085

Echavarria, M. V. (2010). "Problem-Based Learning Application in Engineering”. Revista EIA, (14), 85-95.

Gorbaneff, Y. (2010). “Qué se puede aprender de la literatura sobre el aprendizaje basado en problemas". Revista de la Facultad de Ciencias Económicas: Investigación y Reflexión, 18(1), 61-74.

Kenney, S. S. (2012). Reinventing Undergraduate Education: A Blueprint for America's Research Universities. Illinois: The Boyer Commission on Educating Undergraduates in the Research University/Northern Illinois University.

Morales, P. y V. Landa (2004). “Aprendizaje basado en problemas". Theoria, 13(1), 145-157.

Sánchez S., I. R. y F. J. Ramis (2004). "Aprendizaje significativo basado en problemas". Horizontes Educacionales, (9), 101-111.

Secretaría de Salud (2014). Seguridad vial: Programa de Acción Específico 2013-2018. Programa Sectorial de Salud. México.

STCONAPRA (2011). Manual de Orientaciones Básicas. México: Secretaría de Salud/ Secretariado Técnico del Consejo Nacional para la Prevención de Accidentes. (2017). Seguimiento de indicadores: Indicadores Estratégicos de los Programas de Acción Específicos (PAE). https://drive.google.com/file/d/1OlpMXQH24X6RcJ9PHgRJ9g7lW20WYg FT/vie

(2018). Informe sobre la Situación de la Seguridad Vial, México 2017. México: Secretaría de Salud/Secretariado Técnico del Consejo Nacional para la Prevención de Accidentes. 